\title{
Neurointensive care of traumatic brain injury in the elderly-age-specific secondary insult levels and optimal physiological levels to target need to be defined
}

\author{
Samuel Lenell ${ }^{1} \mathbb{D} \cdot$ Anders Lewén $^{1} \cdot$ Timothy Howells $^{1} \cdot$ Per Enblad $^{1}$
}

Received: 4 August 2021 / Accepted: 25 October 2021 / Published online: 10 November 2021

(C) The Author(s) 2021

\begin{abstract}
Background Elderly patients with traumatic brain injury increase. Current targets and secondary insult definitions during neurointensive care (NIC) are mostly based on younger patients. The aim was therefore to study the occurrence of predefined secondary insults and the impact on outcome in different ages with particular focus on elderly.

Methods Patients admitted to Uppsala 2008-2014 were included. Patient characteristics, NIC management, monitoring data, and outcome were analyzed. The percentage of monitoring time for $I C P, C P P, M A P$, and $S B P$ above-/below-predefined thresholds was calculated.

Results Five hundred seventy patients were included, 151 elderly $\geq 65$ years and 419 younger $16-64$ years. Age $\geq 65$ had significantly higher percentage of $C P P>100, M A P>120$, and $S B P>180$ and age 16-64 had higher percentage of $I C P \geq 20$, $C P P \leq 60$, and $M A P \leq 80$. Age $\geq 65$ contributed independently to the different secondary insult patterens. When patients in all ages were analyzed, low percentage of CPP $>100$ and SBP $>180$, respectively, was significant predictors of favorable outcome and high percentage of $I C P \geq 20, C P P>100, S B P \leq 100$, and $S B P>180$, respectively, was predictors of death. Analysis of age interaction showed that patients $\geq 65$ differed and had a higher odds for favorable outcome with large proportion of good monitoring time with $S B P>180$.

Conclusions Elderly $\geq 65$ have different patterns of secondary insults/physiological variables, which is independently associated to age. The finding that SBP $>180$ increased the odds of favorable outcome in the elderly but decreased the odds in younger patients may indicate that blood pressure should be treated differently depending on age.
\end{abstract}

Keywords Traumatic brain injury $\cdot$ Elderly $\cdot$ Outcome $\cdot$ Secondary insults $\cdot$ Geriatric neurointensive care $\cdot$ Neurointensive care monitoring

\begin{tabular}{lll} 
Abbreviations & EDH & Epidural hematoma \\
ASDH Acute subdural hematoma & EVD & External ventricular drainage \\
CPP Cerebral perfusion pressure & GCS M & Glasgow coma scale motor score \\
CPPopt Optimal cerebral perfusion pressure & GMT & Good monitoring time \\
CSF Cerebrospinal fluid & \%GT & Proportion of good monitoring time \\
CVD Cardiovascular disease & GOSE & Glasgow outcome scale \\
CVP Central venous pressure & ICP & Intra cranial pressure \\
DAI Diffuse axonal injury & MAP & Mean arterial pressure \\
& NIC & Neurointensive care \\
This article is part of the Topical Collection on Neurosurgical & OR & Odds ratio \\
intensive care & SBP & Systolic blood pressure \\
\hline $\begin{array}{l}\text { Samuel Lenell } \\
\text { samuel.lenell @ neuro.uu.se }\end{array}$ & TBI & Traumatic brain injury \\
$\begin{array}{l}\text { Department of Neuroscience/Neurosurgery, Section } \\
\text { of Neurosurgery, Uppsala University, Uppsala University }\end{array}$ & & \\
Hospital, 751 85 Uppsala, Sweden & &
\end{tabular}




\section{Introduction}

The introduction of neurointensive care (NIC), with focused efforts of avoiding secondary insults, has contributed to an increase of favorable outcome for traumatic brain injury (TBI) patients [2, 3, 8, 23, 27]. Despite this improvement, TBI still constitutes a large health problem. The magnitude of the problem is illustrated by a recent overview of TBI in Europe showing that the incidence of hospitalized TBI patients was 278.2/100 000 in 2012 (Sweden 2013, $451.5 / 100000)$ and the mortality rate was $11.7 / 100000$ (Sweden 2013, 9.0/100 000) [21]. Despite that elderly (age $\geq 65$ years) constituted only $29 \%$ of the hospitalized TBI patients, they contributed to $55 \%$ of the mortality [21]. It is obvious that the management of elderly TBI patients will be a tremendous challenge for the future for many reasons. In addition to higher mortality rate in the elderly [10, 17, 21], the elderly are an increasing part of the population and they live more active lives than before $[10,17,18]$. Traditionally, there has been some reluctance to treat these patients due to the previous experience of bad outcome, but more recently, larger numbers of elderly are treated [25, 30, 32, 33, 38]. Hence, it is urgent to obtain more knowledge about the optimal treatment of elderly TBI patients.

The NIC of patients with TBI in general is mostly based on data from younger patients and there is insufficient research in the elderly despite the change in population structure [9]. For example, large clinical TBI trials have often been made with age $>65$ years as an exclusion criteria $[5,14,19,24,26]$. Although the secondary insult prevention concept is one of the main reasons for the improvement of NIC, it is likely that both critical and optimal threshold levels differ between ages. This is underlined by studies in elderly patients with severe subarachnoid hemorrhage showing that the occurrence of defined secondary insults and the impact on outcome was agedependent [31]. In order to optimize the NIC of elderly TBI patients, it is desirable to identify the critical threshold levels for secondary insults and the optimal threshold levels to target, specifically in the older ages.

The aim of this investigation was therefore to study the occurrence of predefined secondary insults and the impact of outcome in different ages with particular focus on the elderly.

\section{Material and methods}

\section{Patient selection and data collection}

All TBI patients $\geq 16$ years old receiving NIC at Uppsala University Hospital between 2008 and 2014 were retrieved from the Uppsala TBI registry [28]. In total, 663 patients were identified. The following patients were excluded as follows: recovery within $24 \mathrm{~h}$ after admission (11 patients), admission more than 5 days after trauma (23 patients), bilateral wide and unresponsive pupils (15 patients) or Glasgow coma scale score 3 and one wide pupil on admission (1 patient) (patients with probable predestined fatal/unfavorable clinical course judged in general not possible to treat $[1,4])$, gunshot to head (4) and lost to follow up (39 patients). Finally, 570 patients remained to be analyzed.

\section{Demographics and NIC management data}

Demographic data and information about NIC management were obtained from the Uppsala TBI registry [28]. The following parameters were studied as follows: age, sex, primary or secondary transfer, Glasgow coma scale motor score (GCS M) on admission, type of injury, presence of multiple injuries, trauma under the influence of alcohol or drugs, cause of trauma, medical history (brain injury/disease, previous traumatic brain injury, diabetes mellitus, hypertension/ cardiovascular disease (CVD), use of anticoagulants/antiplatelets), craniotomy, decompressive craniectomy, intracranial pressure monitoring, and mechanical ventilation. The type of injury was assessed on the initial CT-scan (dominating type of injury and Marshall CT score [22]).

\section{Physiological data}

Trended minute-by-minute data (median values of 5 samples during each sampled minute) was collected in real time from the Philips monitors in our ICU using the Odin software [12]. The Philips monitors forward the data to a central database within the hospital, which is queried by the Odin server to extract the relevant data which is stored centrally and displayed on Odin client systems at the ICU bedspaces. The patient data stored and processed by the Odin software is also kept within the hospital firewall. The trended data used in this study were preprocessed with median filters to detect sudden spikes that appeared to be non-physiological, and a specialized algorithm detected sudden drops to a constant value (usually zero). The data were further subject to manual review to verify, and if necessary correct, the automatic procedures. Time gaps from, e.g., radiology examination and surgical procedure were automatically excluded by the Odin software. The monitoring time left was defined as good monitoring time (GMT).

For the purpose of evaluating physiological NIC monitoring data (intra cranial pressure, ICP; cerebral perfusions pressure, $C P P$; mean arterial pressure, $M A P$; and systolic 
blood pressure, SBP), GMT data from the start of monitoring to the end of the seventh monitoring day was studied.For ICP and $C P P$ analyses, at least $12 \mathrm{~h}$ of $I C P$ data was required. Using the Odin software, the proportions of good monitoring time (\%GMT) spent above-/below-predefined threshold levels were calculated for $I C P \geq 20, C P P \leq 60, C P P>100$, $M A P \leq 80, M A P>120, S B P \leq 100$, and $S B P>180$. The thresholds originated mainly from our protocol treatment goals [8].

\section{Neurointensive care protocol}

All patients were treated according to the same standardized treatment protocol [8]. Unconscious patients (GCS $\mathrm{M} \leq 5$ ) had mechanical ventilation. Patients on mechanical ventilation were kept sedated with propofol (PropofolLipuroB; Braun Medical, Danderyd, Sweden) and received morphine for analgesia. They were initially moderately hyperventilated $\left(\mathrm{PaCO}_{2} 4.0-4.5 \mathrm{kPa}\right)$ with the aim of normoventilation as soon as ICP allowed $(I C P<20 \mathrm{mmHg})$. Wake-up tests were performed regularly (usually 3-6 times/day unless severe ICP elevations) to assess neurological function. All unconscious patients (GCS $M \leq 5$ ), regardless of age, had also ICP monitoring, except in the case of coagulopathy. An external ventricular drainage system (EVD) (with the pressure dome at the level of the lateral ventricles) was the first choice and an intraparenchymal pressure device was chosen if the ventricles were compressed. Arterial blood pressure was measured with the pressure dome at heart level. Prophylactic anticonvulsants was not used. The treatment goals according to the standardized management protocol were as follows: $I C P<20 \mathrm{mmHg}, S B P>100 \mathrm{mmHg}$, central venous pressure (CVP) 0-5 $\mathrm{mmH}_{2} \mathrm{O}, C P P>60 \mathrm{mmHg}$, blood glucose 5-10 mmol/L, normovolemia, $\mathrm{Pa}_{2}>12 \mathrm{kPa}$, electrolytes within normal ranges, and body temperature $<38^{\circ} \mathrm{C}$.

Mass lesions in unconscious patients were evacuated.

Raised ICP was treated in a stepwise fashion. If ICP increased $\geq 20 \mathrm{mmHg}$ without mass lesions, cerebrospinal fluid (CSF) was drained from the EVD. Initially small volumes (1-2 ml) were drained intermittently, when there were risk of expanding hematomas and brain swelling. Later CSF was drained using an open system against a pressure level of $15-20 \mathrm{mmHg}$ if needed. If raised ICP persisted, the treatment was escalated with no wake-up tests, continuous sedation with propofol, and stress reduction with $\beta 1$-antagonist metoprolol (Seloken ${ }^{\circledR}$, AstraZeneca AB Södertälje, Sweden) $(0.2-0.3 \mathrm{mg} / \mathrm{kg} / 24 \mathrm{~h}$ as an infusion) and $\alpha 2$-agonist clonidin (Catapresan ${ }^{\circledR}$, BoehingerIngelheim AB Stockholm Sweden) $(0.5-1.0 \mu \mathrm{g} / \mathrm{kg} \times 8$ or the same dose as an infusion). Thiopental coma treatment and/or decompressive craniectomy were last tier treatment option but were initiated more restrictively in the elderly.

\section{Outcome}

The NIC mortality was assessed. Follow-up was done after 6 months, using the extended Glasgow outcome scale (GOSE), by structured telephone interviews done by a few selected persons[34, 39].

\section{Statistics}

Differences in the characteristics between age groups were analyzed with Pearsons Chi 2 test.

Mann-Withney $U$ test was used to compare occurrence of secondary insults between the age groups.

Multiple linear regression analysis was done to examine if age $\geq 65$ years and admission variables as gender, GCS $\mathrm{M}$, other injuries, extracerebral hematoma, and contusions contributed to the \%GMT above/below secondary insult thresholds for the physiological variables.

To evaluate if the \%GMT above/below secondary insult thresholds for the physiological variables was associated with outcome, univariate logistic regression analysises were made with favorable outcome (GOSE 5-8) and survival (GOSE 2-8) as dependent variables. To evaluate whether associations differed by age (age 16-64 vs age $\geq 65$ ), multiple logistic regression models were fitted including age, a physiological variable and age by physiological variable interaction as independent variables. The odds ratios (ORs) for physiological variables are reported for each age-group, regardless of the significance of interaction.

$p<0.05$ was considered statistically significant. All statistical analyses were carried out in IBM SPSS Statistics for Windows except for Pearsons Chi 2 which was done with Microsoft Excel 365.

\section{Results}

\section{Admission characteristics}

For all patients, the mean age was 49.7 years (range 16-94). The age distribution showed one peak at around 20 years of age and another peak around 60-65 years of age (Supplementary Information 1). There were 151 patients $\geq 65$ years (mean 72.3 range 65-87) and 419 between 16 and 64 (mean 41.5 range 16-64) years of age. Patient characteristics from admission are presented in Table 1. When the age groups of $\geq 65$ years and 16-64 years were compared, the older patients showed significantly larger proportions of women ( $28.5 \%$ vs $19.6 \%$ ), fall accidents ( 80.1 vs $42.0 \%$ ), previous brain injury/disease (22.5\% vs $11.0 \%)$, diabetes mellitus (18.5 vs $6.2 \%$ ), hypertension/cerebrovascular disease ( $58.3 \%$ vs 
Table 1 Patient characteristics by age group

\begin{tabular}{|c|c|c|c|c|c|c|c|}
\hline & \multicolumn{2}{|l|}{ All } & \multicolumn{2}{|c|}{ Age $16-64$} & \multicolumn{2}{|c|}{ Age $\geq 65$} & \multirow{2}{*}{$\begin{array}{l}\text { Age } 16-64 \text { vs } \geq 65^{\circ} \\
p\end{array}$} \\
\hline & $n$ & $\%$ & $n$ & $\%$ & $n$ & $\%$ & \\
\hline No. of patients & 570 & & 419 & & 151 & & \\
\hline Referrals from other hospitals & 447 & 78.4 & 345 & 82.3 & 102 & 67.5 & $<0.001 * * *$ \\
\hline Sex (female) & 125 & 21.9 & 82 & 19.6 & 43 & 28.5 & $0.023 *$ \\
\hline GCS $M \geq 4$ on admission & 518 & 90.9 & 382 & 91.2 & 136 & 90.1 & 0.687 \\
\hline GCS $M \leq 5$ on admission & 310 & 54.4 & 233 & 55.6 & 77 & 51.0 & 0.329 \\
\hline Multiple injuries & 224 & 39.3 & 197 & 47.0 & 27 & 17.9 & $<0.001 * * *$ \\
\hline $\begin{array}{l}\text { Under the influence of drugs/alcohol } \\
\text { at trauma (confirmed) }\end{array}$ & 165 & 28.9 & 143 & 34.1 & 22 & 14.6 & $<0.001 * * *$ \\
\hline \multicolumn{8}{|l|}{ Cause of trauma } \\
\hline Bicycle accident & 16 & 2.8 & 14 & 3.3 & 2 & 1.3 & \\
\hline Fall accident & 297 & 52.1 & 176 & 42.0 & 121 & 80.1 & $<0.001 * * *$ \\
\hline Vehicle accident & 150 & 26.3 & 139 & 33.2 & 11 & 7.3 & $<0.001 * * *$ \\
\hline Pedestrian hit by vehicle & 24 & 4.2 & 17 & 4.1 & 7 & 4.6 & 0.762 \\
\hline Assault & 33 & 5.8 & 30 & 7.2 & 3 & 2.0 & 0.020 \\
\hline Sports injury & 19 & 3.3 & 18 & 4.3 & 1 & 0.7 & $0.033 *$ \\
\hline Other & 31 & 5.4 & 25 & 6.0 & 6 & 4.0 & 0.355 \\
\hline \multicolumn{8}{|l|}{ Medical history } \\
\hline Brain injury/disease previously & 80 & 14.0 & 46 & 11.0 & 34 & 22.5 & $<0.001 * * *$ \\
\hline Traumatic brain injury previously & 18 & 3.2 & 11 & 2.6 & 7 & 4.6 & \\
\hline Diabetes mellitus & 54 & 9.5 & 26 & 6.2 & 28 & 18.5 & $<0.001 * * *$ \\
\hline Hypertension/CVD & 146 & 25.6 & 58 & 13.8 & 88 & 58.3 & $<0.001 * * *$ \\
\hline Anticoagulants/Antiplatelets & 98 & 17.2 & 33 & 7.9 & 65 & 43.0 & $<0.001 * * *$ \\
\hline Ethylism & 126 & 22.1 & 95 & 22.7 & 31 & 20.5 & 0.586 \\
\hline
\end{tabular}

aPearsons Chi 2 test, ${ }^{*} p<0.05, * * p<0.01$, and $* * * p<0.001$
$13.8 \%$ ), ongoing treatment with anticoagulants/antiplatelets $(43.0 \%$ vs $7.9 \%)$, and significantly smaller proportions of patients admitted from other hospitals $(67.5 \%$ vs $82.3 \%$ ), multiple injuries (17.9\% vs $47.0 \%$ ), influence of drugs/alcohol (14.6\% vs $34.1 \%$ ), vehicle accidents (7.3\% vs $33.2 \%$ ), and sports injury ( $0.7 \%$ vs $4.3 \%$ ). Regarding the dominating type of injury assessed on initial CT, the older patients had significantly larger proportion of acute subdural hematoma (51.7\% vs $20.5 \%)$ and smaller proportion of diffuse axonal injury (DAI) $(0.0 \%$ vs $8.6 \%)$ and epidural hematoma $(0.7 \%$ vs $11.5 \%)$ (Table 2$)$. There was no difference between the age groups in GCS M on admission (Table 1 and Supplementary Information 2).

\section{NIC management and surgery}

There were no significant differences between the age groups $\geq 65$ years and 16-64 years regarding ICP monitoring $(55.0 \%$ vs $62.5 \%)$ and mechanical ventilation $(82.1 \%$ vs $77.3 \%$ ) (Table 3). The proportion of patients treated with thiopental were significantly smaller in the old age group $(0.7 \%$ vs $7.9 \%)$ (Table 3$)$. The old group had significantly more craniotomies compared to the younger group (47.7\% vs $32.7 \%$ ) (Table 3).

\section{Physiological data}

Monitoring information regarding number of patients for each physiological parameter and age group is presented in Table 4 . When the occurrences of physiological variables were analyzed as median \%GMT (Table 5 and Fig. 1), there were statistically significant differences between the age groups: age $\geq 65$ years had significantly higher $\% G M T$ with $C P P>100, M A P>120$, and $S B P>180$ and age $16-64$ years had significantly higher $\% G M T$ with $I C P \geq 20, C P P \leq 60$, and $M A P \leq 80$.

The multiple linear regression model with physiological variables as dependent variables and age $\geq 65$ years, gender, GCS M, other injuries, extracerebral hematoma, and contusions as explanatory variables showed that age $\geq 65$ years was an independent predictor for lower \% GMT with $I C P \geq 20$ and higher \%GMT with $C P P>100$, $M A P>120$, and $S B P>180$ (Table 6). Higher GCS M score was an independent predictor for low \%GMT with 
Table 2 Radiological characteristics by age group
Table 3 Treatment characteristics by age group

\begin{tabular}{|c|c|c|c|c|c|c|c|}
\hline & \multicolumn{2}{|l|}{ All } & \multicolumn{2}{|c|}{ Age $16-64$} & \multicolumn{2}{|c|}{ Age $\geq 65$} & \multirow{2}{*}{$\begin{array}{l}\text { Age } 16-64 \text { vs } \geq 65 \\
p\end{array}$} \\
\hline & $n$ & $\%$ & $N$ & $\%$ & $n$ & $\%$ & \\
\hline No. of patients & 570 & & 419 & & 151 & & \\
\hline \multicolumn{8}{|l|}{ Dominating CT finding } \\
\hline ASDH & 164 & 28.8 & 86 & 20.5 & 78 & 51.7 & $<0.001 * * *$ \\
\hline $\mathrm{EDH}$ & 49 & 8.6 & 48 & 11.5 & 1 & 0.7 & $<0.001 * * *$ \\
\hline Contusions & 171 & 30.0 & 132 & 31.5 & 39 & 25.8 & 0.192 \\
\hline DAI & 36 & 6.3 & 36 & 8.6 & 0 & 0.0 & $<0.001 * * *$ \\
\hline Mixed & 68 & 11.9 & 53 & 12.6 & 15 & 9.9 & 0.378 \\
\hline Impression fracture & 12 & 2.1 & 11 & 2.6 & 1 & 0.7 & \\
\hline Traumatic SAH & 53 & 9.3 & 38 & 9.1 & 15 & 9.9 & 0.754 \\
\hline Normal & 6 & 1.1 & 6 & 1.4 & 0 & 0.0 & \\
\hline Other & 11 & 1.9 & 9 & 2.1 & 2 & 1.3 & \\
\hline \multicolumn{8}{|l|}{ Initial CT Marshall classification } \\
\hline Diffuse injury & 393 & 68.9 & 325 & 77.6 & 68 & 45.0 & $<0.001 * * *$ \\
\hline Diffuse injury I & 6 & 1.1 & 5 & 1.2 & 1 & 0.7 & \\
\hline Diffuse injury II & 279 & 48.9 & 236 & 56.3 & 43 & 28.5 & $<0.001 * * *$ \\
\hline Diffuse injury III & 82 & 14.4 & 69 & 16.5 & 13 & 8.6 & $0.018 *$ \\
\hline Diffuse injury IV & 26 & 4.6 & 15 & 3.6 & 11 & 7.3 & 0.061 \\
\hline Focal mass lesion & 117 & 20.5 & 94 & 22.4 & 23 & 15.2 & 0.060 \\
\hline Evacuated mass lesion & 126 & 22.1 & 69 & 16.5 & 57 & 37.7 & $<0.001 * * *$ \\
\hline Nonevacuated mass lesion & 51 & 8.9 & 25 & 6.0 & 26 & 17.2 & $<0.001 * * *$ \\
\hline
\end{tabular}

${ }^{\text {a Pearsons Chi }} 2$ test, $p<0.05,{ }^{* *} p<0.01$, and ${ }^{* * *} p<0.001$

\begin{tabular}{|c|c|c|c|c|c|c|c|}
\hline & \multicolumn{2}{|l|}{ All } & \multicolumn{2}{|c|}{ Age 16-64 } & \multicolumn{2}{|c|}{ Age $\geq 65$} & \multirow{2}{*}{$\begin{array}{l}\text { Age } 16-64 \text { vs } \geq 65^{\mathrm{a}} \\
p\end{array}$} \\
\hline & $N$ & $\%$ & $n$ & $\%$ & $\mathrm{n}$ & $\%$ & \\
\hline No. of patients & 570 & & 419 & & 151 & & \\
\hline \multicolumn{8}{|l|}{ Surgery } \\
\hline Craniotomy at referring hospital & 50 & 8.8 & 36 & 8.6 & 14 & 9.3 & 0.800 \\
\hline Craniotomy (yes/no) & 209 & 36.7 & 137 & 32.7 & 72 & 47.7 & $0.001 * *$ \\
\hline \multicolumn{8}{|l|}{ Reasons for craniotomy ${ }^{b}$} \\
\hline Extra cerebral hematoma & 167 & 29.3 & 99 & 23.6 & 68 & 45.0 & $<0.001 * * *$ \\
\hline $\mathrm{EDH}$ & 35 & 6.1 & 34 & 8.1 & 1 & 0.7 & $0.001 * *$ \\
\hline ASDH & 120 & 21.1 & 55 & 13.1 & 65 & 43.0 & $<0.001 * * *$ \\
\hline Both (EDH + ASDH) & 12 & 2.1 & 10 & 2.4 & 2 & 1.3 & \\
\hline Contusions & 66 & 11.6 & 52 & 12.4 & 14 & 9.3 & 0.301 \\
\hline Hemicraniectomy & 39 & 6.8 & 34 & 8.1 & 5 & 3.3 & $0.045 *$ \\
\hline Multiple surgeries (yes/no) & 61 & 10.7 & 43 & 10.3 & 18 & 11.9 & 0.572 \\
\hline \multicolumn{8}{|l|}{ Management, NIC } \\
\hline ICP monitoring & 345 & 60.5 & 262 & 62.5 & 83 & 55.0 & 0.103 \\
\hline EVD & 65 & 11.4 & 47 & 11.2 & 18 & 11.9 & 0.816 \\
\hline Intraparenchymal pressure monitor & 206 & 36.1 & 153 & 36.5 & 53 & 35.1 & 0.756 \\
\hline Both & 74 & 13.0 & 62 & 14.8 & 12 & 7.9 & $0.032 *$ \\
\hline Mean days with ICP monitoring & 11.2 & & 11.8 & & 9.2 & & \\
\hline Mechanical ventilation & 448 & 78.6 & 324 & 77.3 & 124 & 82.1 & 0.218 \\
\hline Mean days ventilation & 9.0 & & 9.6 & & 7.4 & & \\
\hline Thiopenthal & 34 & 6.0 & 33 & 7.9 & 1 & 0.7 & $0.001 * *$ \\
\hline Mean days with Thiopenthal & 6.2 & & 6.2 & & 6 & & \\
\hline
\end{tabular}

${ }^{a}$ Pearsons Chi 2 test, $* p<0.05, * * p<0.01$, and $* * * p<0.001$

${ }^{\mathrm{b}}$ Multiple operations in some patients 
Table 4 Monitoring by age group

\begin{tabular}{|c|c|c|c|c|c|c|}
\hline & \multirow{2}{*}{$\begin{array}{l}\text { All } \\
N\end{array}$} & \multicolumn{2}{|c|}{ Age 16-64 } & \multicolumn{2}{|c|}{ Age $\geq 65$} & \multirow{2}{*}{$\begin{array}{l}16-64 \text { vs } \geq 65^{\mathrm{a}} \\
p\end{array}$} \\
\hline & & $n$ & $\%$ & $n$ & $\%$ & \\
\hline No. of patients & 570 & 419 & & 151 & & \\
\hline$I C P$ & 333 & 253 & 60.38 & 80 & 52.98 & 0.114 \\
\hline$C P P$ & 332 & $252^{\mathrm{e}}$ & 60.14 & 80 & 52.98 & 0.126 \\
\hline$M A P$ & 521 & 377 & 89.98 & 144 & 95.36 & $0.043 *$ \\
\hline$S B P$ & 521 & 377 & 89.98 & 144 & 95.36 & $0.043 *$ \\
\hline
\end{tabular}

${ }^{a}$ Pearsons Chi 2 test, ${ }^{*} p<0.05$

${ }^{\mathrm{e}}$ Continuous MAP data was missing in one patient with ICP monitoring

Table 5 Occurrence of secondary insults by age group

\begin{tabular}{|c|c|c|c|c|c|c|c|}
\hline \multirow{2}{*}{$\begin{array}{l}\text { Physiological } \\
\text { parameter }\end{array}$} & \multicolumn{2}{|l|}{ All patients } & \multicolumn{2}{|l|}{ Age 16-64 } & \multicolumn{2}{|l|}{ Age $\geq 65$} & \multirow{2}{*}{$\begin{array}{l}16-64 \text { vs } \geq 65^{\mathrm{d}} \\
p\end{array}$} \\
\hline & Median \%GMT & $\overline{\mathrm{IQR} \% \mathrm{GMT}^{\mathrm{c}}}$ & Median $\% \mathrm{GMT}^{\mathrm{c}}$ & IQR $\% \mathrm{GMT}^{\mathrm{c}}$ & Median \%GMT & IQR \%GMT ${ }^{c}$ & \\
\hline$I C P \geq 20$ & 5.26 & $1.28-15.46$ & 6.26 & $1.39-17.01$ & 3.14 & $0.73-9.05$ & $0.005 * *$ \\
\hline$C P P \leq 60$ & 4.72 & $1.60-11.02$ & 5.52 & $2.05-11.79$ & 2.51 & $1.16-1.94$ & $0.001 * *$ \\
\hline$C P P>100$ & 1.97 & $0.62-8.10$ & 1.27 & $0.51-5.25$ & 6.37 & $1.96-18.57$ & $0.000 * * *$ \\
\hline$M A P \leq 80$ & 21.92 & $9.63-38.20$ & 23.01 & $10.67-39.49$ & 17.51 & $8.75-32.68$ & $0.040 *$ \\
\hline$M A P>120$ & 0.59 & $0.21-2.52$ & 0.48 & $0.17-1.77$ & 1.31 & $0.36-5.52$ & $0.000 * * *$ \\
\hline$S B P \leq 100$ & 0.75 & $0.25-2.20$ & 0.75 & $0.25-2.39$ & 0.71 & $0.25-1.83$ & 0.499 \\
\hline$S B P>180$ & 2.10 & $0.23-7.81$ & 1.04 & $0.18-4.72$ & 7.53 & $1.54-19.63$ & $0.000 * * *$ \\
\hline
\end{tabular}

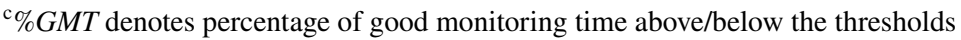

${ }^{\mathrm{d}}$ Mann-Whitney $U$ test, $* p<0.05, * * p<0.01$, and $* * * p<0.001$

Fig. 1 Proportion of good monitoring time $(\% G M T)$ for different insult variables by age group. In the box plots, the horizontal black line marks the median, boxes extend from the 25 th to the 75 th percentile, vertical extending lines denote adjacent values (i.e., the most extreme values within 1.5 interquartile range of the 25th and 75 th percentile of each group) and the dots denote observations outside the range of adjacent values (outliers).Mann-Whitney $U$ test, ${ }^{*} p<0.05, * * p<0.01$, and $* * * p<0.001$

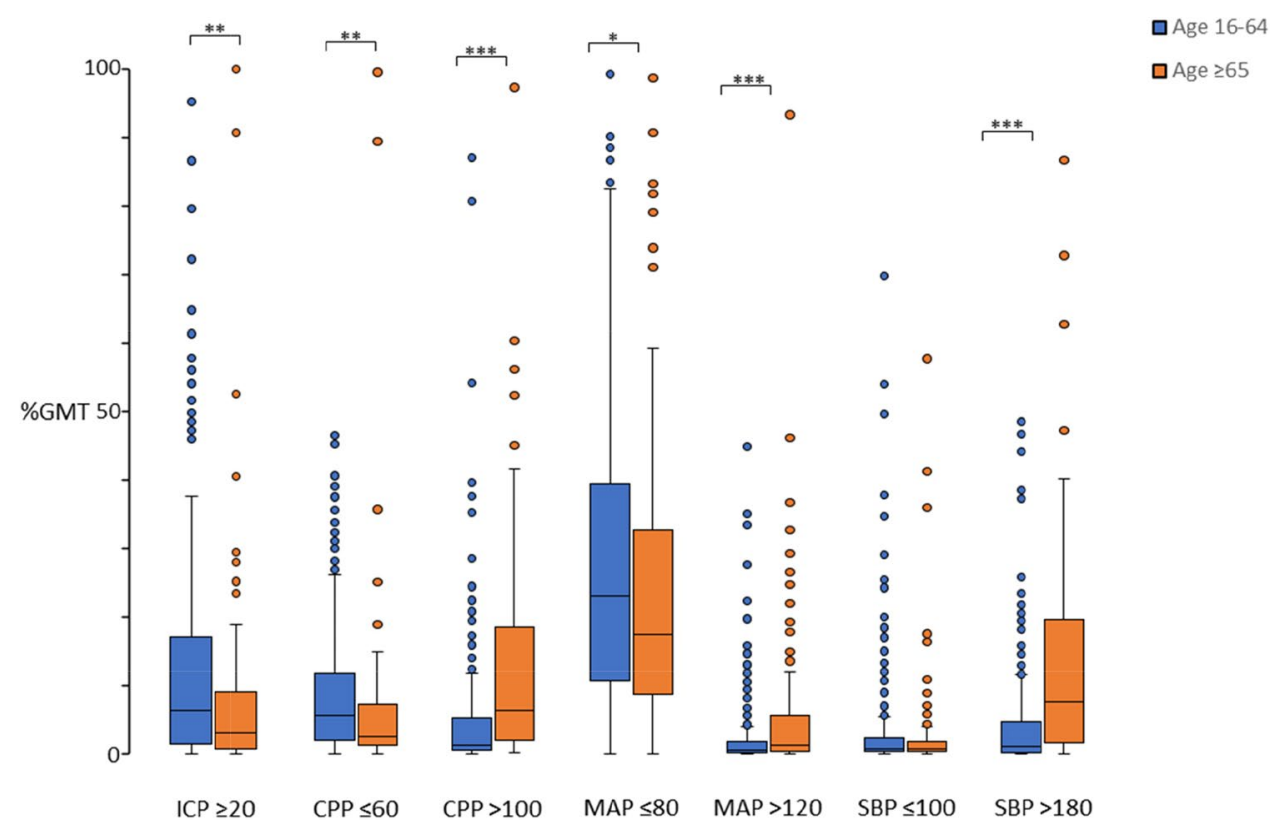

$I C P \geq 20$ and $C P P \leq 60$ (Table 6). Other injuries were found to be an independent predictor for lower \%GMT with $I C P \geq 20, C P P>100, M A P>120$, and $S B P>180$ and for higher $\% G M T$ with $M A P \leq 80$ (Table 6). Females showed significanly lower $\% G M T$ with $S B P>180$ and higher \%GMT with $S B P \leq 100$. (Table 6). 
Table 6 Linear regression analysis of contribution from admission characteristics and age $\geq 65$ to physiological variables

\begin{tabular}{|c|c|c|c|c|c|}
\hline $\begin{array}{l}\text { Physiologi- } \\
\text { cal variable } \\
(\% G M T)\end{array}$ & Explanatory variable & level & $B$ & $(95 \% C I)$ & $p$ value \\
\hline \multirow[t]{6}{*}{$I C P \geq 20$} & Age $\geq 65$ & Yes & -0.05 & $(-0.10$ to -0.10$)$ & $0.016 *$ \\
\hline & Sex (female) & Yes & -0.04 & $(-0.08$ to 0.01$)$ & 0.130 \\
\hline & GCS Motor Score & Per score increase & -0.02 & $(-0.04$ to -0.01$)$ & $0.005 * *$ \\
\hline & Other injuries & Yes & -0.07 & $(-0.04$ to -0.01$)$ & $0.001 * *$ \\
\hline & Extracerebral hematoma & Yes & -0.03 & $(-0.08$ to 0.01$)$ & 0.166 \\
\hline & Contusions & Yes & -0.01 & $(-0.05$ to 0.04$)$ & 0.744 \\
\hline \multirow[t]{6}{*}{$C P P \leq 60$} & Age $\geq 65$ & Yes & -0.02 & $(-0.05$ to 0.01$)$ & 0.176 \\
\hline & Sex (female) & Yes & 0.01 & $(-0.02$ to 0.04$)$ & 0.594 \\
\hline & GCS Motor Score & Per score increase & -0.01 & $(-0.02$ to 0.00$)$ & $0.046 *$ \\
\hline & Other injuries & Yes & 0.00 & $(-0.03$ to 0.02$)$ & 0.836 \\
\hline & Extracerebral hematoma & Yes & -0.01 & $(-0.04$ to 0.03$)$ & 0.687 \\
\hline & Contusions & Yes & -0.01 & $(-0.04$ to 0.02$)$ & 0.486 \\
\hline \multirow[t]{6}{*}{$C P P>100$} & Age $\geq 65$ & Yes & 0.06 & (0.03 to 0.09$)$ & $0.000 * * *$ \\
\hline & Sex (female) & Yes & 0.00 & $(-0.04$ to 0.03$)$ & 0.846 \\
\hline & GCS Motor Score & Per score increase & 0.00 & $(-0.01$ to 0.02$)$ & 0.610 \\
\hline & Other injuries & Yes & -0.05 & $(-0.08$ to -0.02$)$ & $0.000 * * *$ \\
\hline & Extracerebral hematoma & Yes & 0.01 & $(-0.02$ to 0.05$)$ & 0.469 \\
\hline & Contusions & Yes & -0.03 & $(-0.07$ to -0.00$)$ & 0.043 \\
\hline \multirow[t]{6}{*}{$M A P \leq 80$} & Age $\geq 65$ & Yes & -0.02 & $(-0.06$ to 0.02$)$ & 0.347 \\
\hline & Sex (female) & Yes & 0.06 & (0.01 to 0.10$)$ & $0.011 *$ \\
\hline & GCS Motor Score & Per score increase & -0.01 & $(-0.02$ to 0.01$)$ & 0.363 \\
\hline & Other injuries & Yes & 0.09 & $(0.05$ to 0.13$)$ & $0.000 * * *$ \\
\hline & Extracerebral hematoma & Yes & 0.01 & $(-0.04$ to 0.05$)$ & 0.809 \\
\hline & Contusions & Yes & 0.00 & $(-0.05$ to 0.05$)$ & 0.939 \\
\hline \multirow[t]{6}{*}{$M A P>120$} & Age $\geq 65$ & Yes & 0.02 & (0.01 to 0.04$)$ & $0.009 * *$ \\
\hline & Sex (female) & Yes & 0.00 & $(-0.02$ to 0.01$)$ & 0.828 \\
\hline & GCS Motor Score & Per score increase & 0.00 & (0.00 to 0.008$)$ & 0.522 \\
\hline & Other injuries & Yes & -0.03 & $(-0.04$ to -0.02$)$ & $0.000 * * *$ \\
\hline & Extracerebral hematoma & Yes & 0.00 & $(-0.02$ to 0.02$)$ & 0.964 \\
\hline & Contusions & Yes & 0.00 & $(-0.02$ to 0.01$)$ & 0.636 \\
\hline \multirow[t]{6}{*}{$S B P \leq 100$} & Age $\geq 65$ & Yes & -0.01 & $(-0.02$ to 0.01$)$ & 0.364 \\
\hline & Sex (female) & Yes & 0.02 & (0.01 to 0.04$)$ & $0.001 * *$ \\
\hline & GCS Motor Score & Per score increase & 0.00 & $(-0.01$ to 0.00$)$ & 0.116 \\
\hline & Other injuries & Yes & -0.01 & $(-0.02$ to 0.00$)$ & 0.119 \\
\hline & Extracerebral hematoma & Yes & 0.00 & $(-0.02$ to 0.01$)$ & 0.732 \\
\hline & Contusions & Yes & -0.01 & $(-0.02$ to 0.01$)$ & 0.455 \\
\hline \multirow[t]{6}{*}{$S B P>180$} & Age $\geq 65$ & Yes & 0.08 & (0.06 to 0.12$)$ & $0.000 * * *$ \\
\hline & Sex (female) & Yes & -0.03 & $(-0.05$ to -0.01$)$ & $0.001 * *$ \\
\hline & GCS Motor Score & Per score increase & 0.00 & $(-0.01$ to 0.01$)$ & 0.474 \\
\hline & Other injuries & Yes & -0.03 & $(-0.05$ to -0.01$)$ & $0.002 * *$ \\
\hline & Extracerebral hematoma & Yes & 0.00 & $(-0.03$ to 0.02$)$ & 0.745 \\
\hline & Contusions & Yes & 0.01 & $(-0.02$ to 0.03$)$ & 0.657 \\
\hline
\end{tabular}

Multivariate linear regression analyses of each physiological variables as dependent and age $\geq 65$, sex, GCS motor score, other injuries, extracerebral hematoma, and contusions as explanatory variables. $* p<0.05$, $* * p<0.01$, and $* * * p<0.001$. Positive $B$ coefficients indicate that the increasing value of the explanatory variable are associated with a larger \%GMT of the dependent variable. Negative $B$ coefficients indicate that the increasing value of the explanatory variable are associated with a lower \%GMT of the dependent variable 


\section{Outcome}

NIC mortality was higher in the old age group ( $\geq 65$ years $8.6 \%$ and $16-64$ years $2.4 \%, p<0.001$ ). Follow-up was made at 7 months in median (range 1-28, including patients who died before follow-up). For all ages, favorable outcome (GOSE 5-8) was observed in 62\% (69\% in 16-64 years and $42 \%$ in elderly) and $13 \%$ had died (6\% in 16-64 years and $31 \%$ in elderly) (Fig. 2).

The results from the logistic regression analyses with favorable outcome and survival as dependent variables and physiological parameters as explanatory variables are presented in Table 7. Low \%GMT with $C P P>100$ and $S B P>180$ were associated with a higher odds of favorable outcome. However, there was a statistically significant interaction between age and \%GMT with $S B P>180(p$ interaction $=0.025$ ). The $O R$ (per unit increase in $\% G M T$ with $S B P>180)$ was $2.07(0.22-1731.66)$ in patients $\geq 65$ years and $-0.03(0.00-0.57)$ in patients 16-64 years (Table 7). High $\% G M T$ with $I C P \geq 20, C P P>100, S B P \leq 100$ were associated with a lower odds of survival (Table 7).

\section{Discussion}

\section{Patient and management characteristics by age group}

Patients $\geq 65$ years of age constituted as much as $26 \%$ of all patients. Many of the patient characteristics found in relation to age were as expected. The most common cause of trauma in the elderly was fall accidents, which is in accordance with many other studies $[7,11,13,15,16,18,20,29$, $35,36]$. There was a higher percentage of women among the elderly (29\% vs 20\%), which also was shown by DamsO'Conner and coll., reporting an increasing proportion of women with increasing age (38.5\% in 65-74 years, $50.4 \%$ in 75-84 years, and 62.2 in 85 years and older) [7]. The elderly more often had a medical history with previous diseases or injuries, e.g., $22.5 \%$ had a previous history of brain injury/disease, $58.3 \%$ hypertension/CVD, and $43 \%$ medicated with anticoagulants/antiplatelets. Similar results were found by Hawley and coll. showing that older TBI patients $\geq 65$ had a recorded medical history in $80 \%$ and only $1.1 \%$ had no pre-existing medical condition [11]. The dominating injury type in the elderly was ASDH and diffuse injury was also less common according to the Marshall score. These findings are in line with that the dominating type of injury was falls in the elderly and that the elderly more often underwent craniotomy.

\section{Secondary insults/physiological variables- occurrence and association to age}

The pattern of secondary insults/physiological variables differed by age. The elderly ( $\geq 65$ years) spent a higher proportion of GMT with high $C P P$, high $M A P$, and high $S B P$ and less degree of high ICP, low $C P P$, and low MAP (Table 5). Similar findings were also observed by Czosnyka and coll. [6].

In order to find out whether the observed difference between the age groups was explained by age independently, a multiple linear regression analysis was performed including age $\geq 65$ years as a explanatory factor for the different predefined secondary insults/physiological variables. The analysis showed that age $\geq 65$ years was an independent explanatory factor for higher \%GMT with $C P P>100$, $M A P>120$, and $S B P>180$ (Table 6). This finding may to some extent be explained by higher degree of hypertension and cardiovascular diseases in the elderly (Table 1). The crucial question is whether higher pressures may influence outcome in a negative way in the elderly.

Fig. 2 Clinical outcome at follow-up. Favorable outcome (GOSE 5-8), unfavorable (GOSE 3-4), vegetative (GOSE 2), and dead (GOSE 1). Each bar represents the percentage of outcome within its age group. Absolute number of patients in each bar is presented above

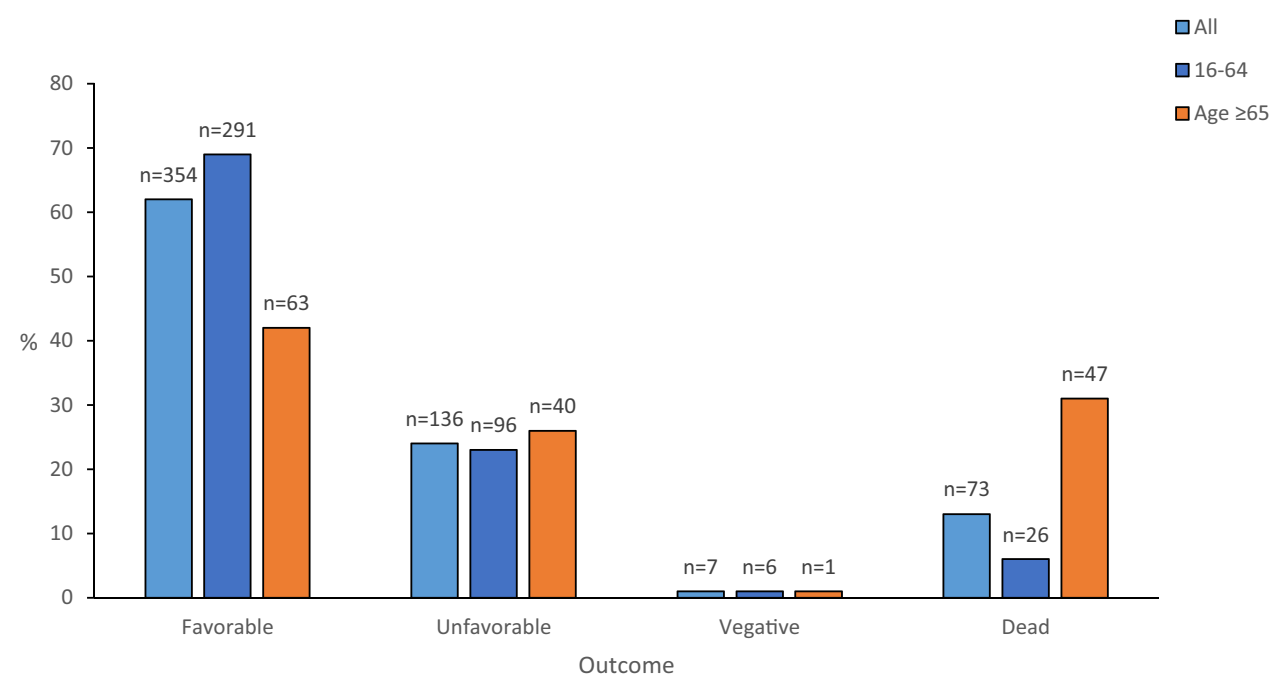




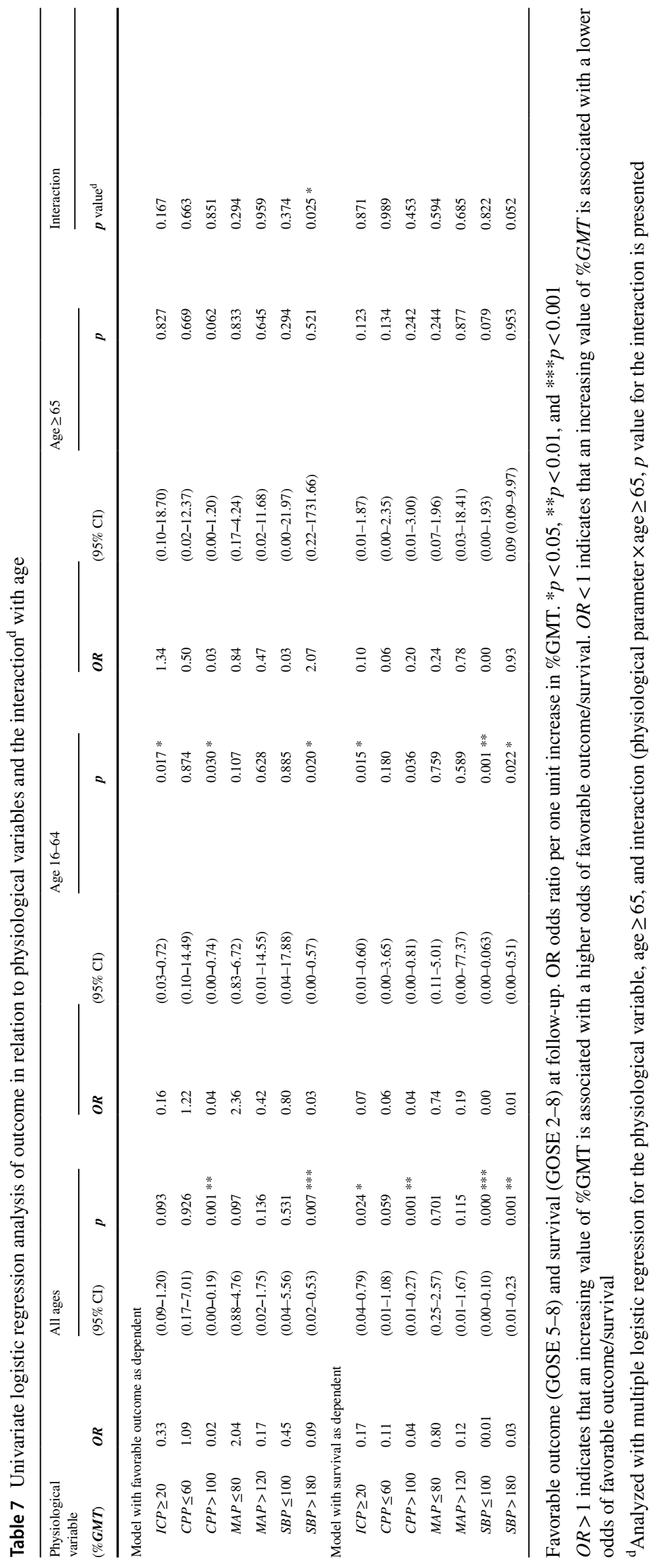




\section{Secondary insults/physiological variables-relation to clinical outcome and interaction by age}

The logistic regression analysis of outcome (favorable and survival) for all patients indicated that high $\% G M T$ with $I C P>20, S B P \leq 100, S B P>180, C P P>100$ not are beneficial. These findings, which may be summarized roughly as high $I C P$, low and high $\mathrm{BP}$, and high $C P P$ are bad, were not unexpected. Interestingly, when looking at the interaction analyses, the elderly had a higher AOR for favorable outcome.

Hence, blood pressure should probably be treated differently in younger and older patients. The finding that high blood pressures may be advantageous in elderly is supported by Utomo and coll. who found higher odds of independent living at 6 months for patients $\geq 65$ years with a $S B P$ on arrival at hospital in the range of 131-150 $\mathrm{mmHg}$, compared to patients with $S B P$ of $<130 \mathrm{mmHg}$ [37].

ICP did not prove to be a significant predictor of outcome in the elderly. This finding should not be interpreted as if ICP is unimportant for outcome and does not need to be monitored in the elderly. Instead, this is probably an effect of the low burden of ICP insults thanks to effective detection and treatment. We have examined our material for events with $\% G M T I C P \geq 25$ and there was very few events in the elderly (median \%GMT was 0.53 , unpublished data). Monitoring of ICP in elderly with TBI is of importance and this has also been shown by You and coll. in a randomized trial of elderly with severe TBI who found lower in-hospital mortality and improved 6-month outcomes for the patients randomized to ICP monitoring [40]. We belive that extensive NIC monitoring is even more important in the elderly due to their increased vulnerability and this philosophy was clearly reflected in the observed numbers of elderly monitored in this study (Table 4), despite a larger proportion elderly using anticoagulants/antiplatelets.

\section{Limitations}

This is a single-center study and the results may therefore be influenced by the local management applied. Thus, the results may not be completely generalizable. There was a selection bias since only patients judged to have a reasonable chance for favorable outcome were accepted for NIC. Treatment bias also needs to be considered since all patients were treated to avoid secondary insults and the \% GMT at insult level was in low general.

Furthermore, complete multiple logistic regression analyses for assessing the influence of secondary insults on outcome could not be done (to adjust, e.g., sex, GCS at admission, and injury type) due to the relative small number of patients. It was however possible to study the age interaction.

\section{Conclusions}

Elderly $\geq 65$ years have different patterns of secondary insults/physiological variables, which to some extent is independently associated to age. When patients in all ages were analyzed, low \%GMT with $C P P>100$ and $S B P>180$ were significant predictors of favorable outcome and high \%GMT with $I C P \geq 20, C P P>100, S B P \leq 100$, and $S B P>180$ were positive predictors of death. The analysis of age interaction showed that patients $\geq 65$ years differed and had a higher odds for favorable outcome and without a significant decrease in survival with large proportion of good monitoring time with $S B P>180$.

This finding may indicate that blood pressure should be treated differently in younger and older patients. More TBI studies in the elderly are warrented to define specific guidelines regarding secondary insult definitions and optimal levels to target. Studies of pressure autoregulation and CPPopt are also desirable.

Supplementary Information The online version contains supplementary material available at https://doi.org/10.1007/s00701-021-05047-z.

Acknowledgements Mona-Lisa Wernroth at Uppsala Clinical research center, UCR, is acknowledged for statistical expertise advice.

Funding Open access funding provided by Uppsala University. The study was supported by grants from the Uppsala university hospital.

\section{Declarations}

Ethical approval All procedures performed in studies involving human participants were in accordance with the ethical standards of the institutional and/or national research committee and with the 1964 Helsinki declaration and its later amendments or comparable ethical standards. This article does not contain any studies with animals performed by any of the authors. The study was approved by the local ethical review board.

Informed consent Informed consent was obtained from individual participants or the relatives if the participant did not have the decisionmaking capacity for informed consent.

Conflict of interest The authors declare no competing interests.

Open Access This article is licensed under a Creative Commons Attribution 4.0 International License, which permits use, sharing, adaptation, distribution and reproduction in any medium or format, as long as you give appropriate credit to the original author(s) and the source, provide a link to the Creative Commons licence, and indicate if changes were made. The images or other third party material in this article are included in the article's Creative Commons licence, unless indicated otherwise in a credit line to the material. If material is not included in the article's Creative Commons licence and your intended use is not permitted by statutory regulation or exceeds the permitted use, you will need to obtain permission directly from the copyright holder. To view a copy of this licence, visit http://creativecommons.org/licenses/by/4.0/. 


\section{References}

1. Andrews BT, Pitts LH (1991) Functional recovery after traumatic transtentorial herniation. Neurosurgery 29(2):227-231

2. Becker P, Ward JD, Young HF (1977) The outcome from severe head injury with early diagnosis and intensive management. J Neurosurg 4:12

3. Bowers SA, Marshall LF (1980) Outcome in 200 consecutive cases of severe head injury treated in San Diego County: a prospective analysis. Neurosurgery 6(3):237-242

4. Combes R, Fauvage B, Jacquot C, Colonna M, Passagia JG, Chirossel JR, de Grenoble C Severe head injuries: an outcome prediction and survival analysis. 5

5. Cooper DJ, Rosenfeld JV, Murray L et al (2011) Decompressive craniectomy in diffuse traumatic brain injury. N Engl J Med 364(16):1493-1502

6. Czosnyka M, Balestreri M, Steiner L, Smielewski P, Hutchinson PJ, Matta B, Pickard JD (2005) Age, intracranial pressure, autoregulation, and outcome after brain trauma. J Neurosurg 102(3):450-454

7. Dams-O'Connor K, Cuthbert JP, Whyte J, Corrigan JD, Faul M, Harrison-Felix C (2013) Traumatic brain injury among older adults at level I and II trauma centers. J Neurotrauma 30(24):2001-2013

8. Elf K, Nilsson P, Enblad P (2002) Outcome after traumatic brain injury improved by an organized secondary insult program and standardized neurointensive care*: Crit Care Med 30(9):2129-2134

9. Gaastra B, Longworth A, Matta B, Snelson C, Whitehouse T, Murphy $\mathrm{N}$, Veenith $\mathrm{T}$ (2016) The ageing population is neglected in research studies of traumatic brain injury. Br J Neurosurg 30(2):221-226

10. Gardner RC, Dams-O'Connor K, Morrissey MR, Manley GT (2018) Geriatric traumatic brain injury: epidemiology, outcomes, knowledge gaps, and future directions. J Neurotrauma 35(7):889-906

11. Hawley C, Sakr M, Scapinello S, Salvo J, Wrenn P (2017) Traumatic brain injuries in older adults -6 years of data for one UK trauma centre: retrospective analysis of prospectively collected data. Emerg Med J 34(8):509-516

12. Howells T, Piper I, Souter M, Miller J (1995) Jones P Design of a research database for the study of secondary insults following head injury. J Neurotrauma 12:471-472

13. Hukkelhoven CWPM, Steyerberg EW, Rampen AJJ, Farace E, Habbema JDF, Marshall LF, Murray GD, Maas AIR (2003) Patient age and outcome following severe traumatic brain injury: an analysis of 5600 patients. J Neurosurg 99(4):666-673

14. Hutchinson PJ, Kolias AG, Timofeev IS et al (2016) Trial of decompressive craniectomy for traumatic intracranial hypertension. N Engl J Med 375(12):1119-1130

15. Iaccarino C, Carretta A, Nicolosi F, Morselli C (2018) Epidemiology of severe traumatic brain injury. J Neurosurg Sci 62(5):8

16. Koskinen S, Alaranta H (2008) Traumatic brain injury in Finland 1991-2005: a nationwide register study of hospitalized and fatal TBI. Brain Inj 22(3):205-214

17. Lenell S, Nyholm L, Lewén A, Enblad P (2015) Updated periodic evaluation of standardized neurointensive care shows that it is possible to maintain a high level of favorable outcome even with increasing mean age. Acta Neurochir (Wien) 157(3):417-425

18. Lennartsson C, Heimerson I (2012) Elderly people's health: Health in Sweden: the National Public Health Report 2012. Chapter 5. Scand J Public Health 40(9 Suppl):95-120
19. Maas AI, Murray G, Henney H, Kassem N, Legrand V, Mangelus M, Muizelaar J-P, Stocchetti N, Knoller N (2006) Efficacy and safety of dexanabinol in severe traumatic brain injury: results of a phase III randomised, placebo-controlled, clinical trial. Lancet Neurol 5(1):38-45

20. Maas AI, Stocchetti N, Bullock R (2008) Moderate and severe traumatic brain injury in adults. Lancet Neurol 7(8):728-741

21. Majdan M, Plancikova D, Brazinova A, Rusnak M, Nieboer D, Feigin V, Maas A (2016) Epidemiology of traumatic brain injuries in Europe: a cross-sectional analysis. Lancet Public Health 1(2):e76-e83

22. Marshall LF, Marshall SB, Klauber MR, van Clark M, B, Eisenberg HM, Jane JA, Luerssen TG, Marmarou A, Foulkes MA, (1991) A new classification of head injury based on computerized tomography. J Neurosurg 75(Supplement):S14-S20

23. Marshall LF, Smith RW, Shapiro HM (1979) The outcome with aggressive treatment in severe head injuries. Part II: acute and chronic barbiturate administration in the management of head injury. J Neurosurg 50(1):26-30

24. Marshall LF, Maas AIR, Marshall SB et al (1998) A multicenter trial on the efficacy of using tirilazad mesylate in cases of head injury. J Neurosurg 89(4):519-525

25. Merzo A, Lenell S, Nyholm L, Enblad P, Lewén A (2016) Promising clinical outcome of elderly with TBI after modern neurointensive care. Acta Neurochir (Wien) 158(1):125-133

26. Nichol A, French C, Little L et al (2015) Erythropoietin in traumatic brain injury (EPO-TBI): a double-blind randomised controlled trial. The Lancet 386(10012):2499-2506

27. Nordström C-H, Gör Sundbärg, Messeter K, Schalén W (1989) Severe traumatic brain lesions in Sweden. Part 2: impact of aggressive neurosurgical intensive care. Brain Inj 3(3):267-281

28. Nyholm L, Howells T, Enblad P, Lewén A (2013) Introduction of the Uppsala Traumatic Brain Injury register for regular surveillance of patient characteristics and neurointensive care management including secondary insult quantification and clinical outcome. Ups J Med Sci 118(3):169-180

29. Peeters W, Majdan M, Brazinova A, Nieboer D, Maas AIR (2017) Changing epidemiological patterns in traumatic brain injury: a longitudinal hospital-based study in Belgium. Neuroepidemiology 48(1-2):63-70

30. Raj R, Mikkonen ED, Kivisaari R, Skrifvars MB, Korja M, Siironen $\mathrm{J}$ (2016) Mortality in elderly patients operated for an acute subdural hematoma: a surgical case series. World Neurosurg 88:592-597

31. Ryttlefors M, Howells T, Nilsson P, Ronne-Engström E, Enblad $\mathrm{P}$ (2007) Secondary insults in subarachnoid hemorrhage: occurrence and impact on outcome and clinical deterioration. Neurosurgery 61(4):704-714; discussion 714-715

32. Shimoda K, Maeda T, Tado M, Yoshino A, Katayama Y, Bullock MR (2014) Outcome and surgical management for geriatric traumatic brain injury: analysis of 888 cases registered in the Japan Neurotrauma Data Bank. World Neurosurg 82(6):1300-1306

33. Taussky P, Hidalgo ET, Landolt H, Fandino J (2012) Age and salvageability: analysis of outcome of patients older than 65 years undergoing craniotomy for acute traumatic subdural hematoma. World Neurosurg 78(3-4):306-311

34. Teasdale GM, Pettigrew LEL, Wilson JTL, Murray G, Jennett B (1998) Analyzing outcome of treatment of severe head injury: a review and update on advancing the use of the Glasgow Outcome Scale. J Neurotrauma 15(8):587-597

35. Thomas KE, Stevens JA, Sarmiento K (2005) Wald MM (2008) Fall-related traumatic brain injury deaths and hospitalizations among older adults — United States. J Safety Res 39(3):269-272 
36. Thompson HJ, McCormick WC, Kagan SH (2006) Traumatic brain injury in older adults: epidemiology, outcomes, and future implications: traumatic brain injury and older adultS. J Am Geriatr Soc 54(10):1590-1595

37. Utomo WK, Gabbe BJ, Simpson PM, Cameron PA (2009) Predictors of in-hospital mortality and 6-month functional outcomes in older adults after moderate to severe traumatic brain injury. Injury 40(9):973-977

38. Wan X, Liu S, Wang S et al (2016) Elderly patients with severe traumatic brain injury could benefit from surgical treatment. World Neurosurg 89:147-152
39. Wilson JTL, Pettigrew LEL, Teasdale GM (1998) Structured interviews for the Glasgow Outcome Scale and the Extended Glasgow Outcome Scale: guidelines for their use. J Neurotrauma 15(8):573-585

40. You W, Feng J, Tang Q, Cao J, Wang L, Lei J, Mao Q, Gao G, Jiang J (2015) Intraventricular intracranial pressure monitoring improves the outcome of older adults with severe traumatic brain injury: an observational, prospective study. BMC Anesthesiol 16(1):35

Publisher's note Springer Nature remains neutral with regard to jurisdictional claims in published maps and institutional affiliations. 